\title{
Comparison of intradimensional and extradimensional shifts using geometric and symbolic stimuli
}

\author{
THOMAS D. KENNEDY \\ Arizona State University, Tempe, Arizona 85281 \\ and \\ CHARLES D. GERSTEN \\ F.D.R. Veterans Administration Hospital, Montrose, New York 10548
}

\begin{abstract}
In two experiments, college students were required to make intradimensional (ID) or extradimensional (ED) shifts when potential confounding factors were eliminated through the use of a total change design and symbolic (word) stimuli. Results indicated that an ID shift was learned significantly faster than an ED shift, whether geometric or symbolic stimuli were employed. Original learning with both classes of stimuli was found to be comparable. Evidence was lacking for the hypothesis that a nonmediational process, such as primary stimulus generalization, accounted for ID shift superiority. The results were interpreted as supporting a two-stage mediational explanation of human discrimination learning.
\end{abstract}

The basic experimental design for studying human discrimination learning, the shift paradigm, was introduced by Buss (1953) who found that reversal (RV) shifts were easier to make than extradimensional (ED) shifts. Buss reasoned that RV shift superiority was due to the fact that ED subjects experienced fortuitous partial reinforcement of preshift sorting responses during the postshift problem, thereby retarding the acquisition of the new solution. This explanation is subsumed within the single-stage theory of discrimination learning. The theory postulates that associations are formed directly between the discriminative cues on the stimulus cards and the sorting responses.

When intermittent reinforcement of the sorting responses was eliminated during the postshift problem, Kendler and D'Amato (1955) found that an RV shift was still considerably easier than an ED shift. The result was explained in terms of a two-stage mediational theory, which assumes the acquisition of a mediational response during the preshift phase corresponding in some manner to the relevant dimension. Since the mediational response remains appropriate after an RV shift, this type of shift is predicted by mediational theory to be easier than an ED shift due to the fact that the latter shift requires the acquisition of a new mediational response.

Subsequent to these initial investigations,

The authors wish to thank Linda McElwain who served as the experimenter. A brief version of Experiment $I$ was presented at the annual meeting of the Southwestern Psychological Association in San Antonio, April, 1971. Requests for reprints should be sent to Thomas D. Kennedy, who is now at the Center of Criminal Justice, Arizona State University, Tempe, Arizona 85281. numerous studies employing methodological variations for eliminating intermittent reinforcementsuch as the optional shift and the partial change design-were conducted in an attempt to provide theoretical clarification. Extradimensional shifts were consistently found to be more difficult to accomplish than RV shifts. In reviewing these studies, Slamecka (1968) systematically identified other potential sources of bias which could confound the results and complicate the interpretation of the data. To eliminate these biases, Slamecka advocated the use of a total change design in which an RV shift is usually referred to as an intradimensional (ID) shift. This design, which employs new values on all dimensions for all groups during the postshift problem, was used by Eimas (1966) with children and by Gersten (Note 1) with college students. The Eimas and Gersten studies produced results consistent with mediation theory in that ID shifts were performed significantly faster than ED shifts. Slamecka noted, however, that the use of the usual colored geometric stimuli in such studies "might conceivably allow a nonmediational account based upon the operation of a primary stimulus-generalization mechanism (1968, p. 436)." To control for this possibility, Slamecka suggested the use of symbolic stimuli such as printed words.

Using word stimuli and a total change design, Slamecka (1969) found an ID shift to be easier than an ED shift, favoring mediation theory. LeBow and Tritt (1971) noted that the stimuli used by Slamecka (1969) were common nouns such as cities, sports, and animals rather than the dimensions of form, color, and number typically used when colored geometric 
figures serve as stimuli. As a result, LeBow and Tritt used typed words representing the dimensions of form, color, and number (e.g., triangles, yellow, three), and again found that an ID shift was learned faster than an ED shift. In both of these studies, however, no comparison of shift performance with traditional geometric figures as stimuli was made. Nor did LeBow and Tritt (1971) indicate the mean number of trials or errors to criterion during original learning or transfer.

Thus, the present two experiments were conducted to provide data on the relative difficulty of original learning and transfer with geometric and symbolic stimuli, as well as to test further single-stage vs. mediational interpretations of discrimination learning within the total change design.

\section{EXPERIMENT I}

\section{Method}

Subjects. Eighty university undergraduates enrolled in the introductory psychology course were randomly assigned to four groups. Three subjects, each from a different group, who failed to reach solution in 144 trials during the preshift problem were considered nonsolvers and eliminated from the experiment. These subjects were replaced by other students to maintain a balance of 20 subjects per group. None of the subjects professed any prior knowledge of the experiment, nor had any previously participated in a concept identification task. All claimed to have normal color vision.

Stimuli and apparatus. Two decks of colored geometric stimulus cards were composed from the Wisconsin Card Sorting Test. The stimuli varied on two dimensions, form and color, one or the other of which was relevant at some time during the experiment. In addition, the stimuli varied in number, a dimension that was always irrelevant. Each deck of cards consisted of two sets of eight cards, each set representing the eight distinct cue combinations for the three dimensions. The dimensions for one deck (red-blue) were: form-circle, triangle; color-red, blue; number-one or three forms on a card. For the other deck (yellow-green), they were: form-star, cross; color-yellow, green; number-two or four. The sequence of cards in each set of each deck was randomly determined with the exception that no stimulus card succeeded itself in the deck. The cards remained in their prearranged order throughout the experiment and were recycled until the subject reached the criterion of 10 consecutive errorless trials. One deck of symbolic stimulus cards corresponded to the red-blue deck in all respects and the other symbolic deck was the same as the yellow-green deck. On these cards, the appropriate stimulus was printed in black block letters $.8 \mathrm{~cm}$ high (e.g., ONE BLUE CIRCLE).

The subject and the experimenter, seated on opposite sides of a table, were separated by a vertical unpainted masonite panel $61 \mathrm{~cm}$ high $\times 121.9 \mathrm{~cm}$ wide. On the face of the panel were three slots which permitted exchange of the stimulus cards. One slot, centered at the bottom of the panel, was used by the experimenter to present the cards to subjects one at a time. Two slots were positioned in a horizontal line $30.5 \mathrm{~cm}$ from the bottom of the panel. One slot was $30.5 \mathrm{~cm}$ to the right of the centerline and the other was a like distance to the left. These two slots were used by subjects for sorting each card and returning it to the experimenter.

Instructions. Each subject was seen individually. Before taking his seat, the experimenter read the following instructions to the subject: "I have a number of cards like these (subject shown two sample cards) which I am going to give to you one at a time through this bottom slot. Some of the cards belong in this slot (pointing), while others belong in this slot (pointing). Your task will be to find out which cards go into which slot. That is, you are to learn to sort the cards by placing each card in the correct slot. Each time I give you a card, pick the card up without rotating it and push it back to me face up through whichever slot you think is correct. If you place the card in the correct slot, I'll say 'right'; if you place it in the incorrect slot, I'll say 'wrong.' Do you have any questions? (Questions were answered by repeating appropriate parts of the instructions.) The experiment will take about $30 \mathrm{~min}$. Once you begin, you will not be permitted to ask any questions until I have told you that you are finished. Before beginning, do you have any questions? (Pause) Okay, let's begin."

Design and procedure. There were two ID and two ED shift groups. The two groups presented with colored geometric stimulus cards throughout the experiment were designated as Groups IDG and EDG. Groups IDW and EDW received the symbolic (i.e., word) stimulus cards throughout. In the preshift phase of the experiment, half of the subjects in each group were required to sort the cards on the basis of form and half on the basis of color. With respect to each relevant dimension (i.e., form or color), the red-blue deck was used for half the subjects and the yellow-green deck for the remaining half in each group. When subject reached criterion on the preshift problem, the experimenter began the postshift phase without interruption by switching to the other deck of cards (i.e., from the red-blue deck to the yellow-green or the converse). Thus, all subjects were presented with new values on all dimensions during the postshift phase of the experiment.

To solve the postshift problem, subjects in the ED groups had to make an ED shift which involved a change in the relevant dimension from color to form or form to color, depending on the initially relevant dimension. Problem solution for subjects in the ID groups necessitated the making of an ID shift which involved a change in the cue values of the relevant dimension from red-blue to yellow-green (or the converse) or from circle-triangle to star-cross (or the opposite), depending on the preshift relevant cues. This arrangement produced a 2 by 2 by 2 factorial design in which stimulus (geometric vs. symbolic), shift (ID vs. ED), and dimension (form vs. color) were the factors.

\section{Results}

Since raw score trials and errors to criterion did not conform to the $F$ test assumptions of equality of variance and normality, the scores were transformed logarithmically. Transformation eliminated abnormalities of the data. Analyses of variance of the transformed scores on trials and errors showed the same effects. Consequently, only analyses of the transformed trials data are reported.

Preshift mean trials to criterion for Groups IDG, EDG, IDW, and EDW were .997, 1.031, 1.097, and .686 , respectively. Corresponding postshift means were $.440,1.266, .675$, and 1.170 . An analysis of variance of the preshift scores indicated that all main effects and interactions were nonsignificant (all ps $>.10)$. Inspection of the group averages show that the ID groups demonstrated positive transfer to the postshift problem whereas the ED groups demonstrated negative transfer, and that both ID groups required fewer postshift trials to criterion than either of the ED groups.

The analysis of variance of the postshift scores revealed that the Shift effect was significant, $F(1,72)=27.20, p<.01$, indicating that an ID shift was significantly easier than an ED shift. All other main effects and interactions were not statistically 
reliable (all ps $>.10$ ). Of particular interest was the Shift by Stimulus interaction, $F(1,72)=1.72$, $p=.19$. This finding lends no support to the hypothesis that stimulus generalization may be a biasing factor favoring ID shift performance with colored geometric stimuli as proposed by Slamecka (1968).

In addition, the design of the experiment permitted a more direct check of stimulus generalization. That is, during the preshift, the correct stimulus pairings for half the subjects in each ID color subgroup (where color was the relevant dimension throughout the experimental task) were red-left, blue-right; for the remaining half they were yellow-left, green-right. On the postshift problem, the pairings were reversed; yellow-left, green-right, and red-left, blue-right, respectively. Responding in a manner consistent with a generalization gradient on the initial trial of the postshift task, then, would lead to a correct stimulus pairing. Thus, if the subgroup exposed to geometric stimuli made significantly more correct pairings on the first trial of the postshift than the subgroup presented with symbolic materials, stimulus generalization could be considered a plausible explanation. It was found that $40 \%$ of the subjects in the IDG subgroup paired correctly on the first trial, whereas $30 \%$ of the subjects in the IDW subgroup did so. The test of the difference between the two proportions was not reliable, $z=.47, \mathrm{p}>.20$. It was also found that only $10 \%$ of the subjects in both subgroups made consecutive errors on the first two trials of the postshift. It seems clear, then, that subjects presented with geometric stimuli did not begin the postshift task with an initial advantage which might be attributable to stimulus generalization.

\section{EXPERIMENT II}

\section{Method}

The stimuli, apparatus, procedure, and instructions were the same as described for Experiment I. Experiment II differed in that there were 10 subjects per group, and the design employed identical transfer tasks. That is, Groups IDG and IDW began with color as the relevant dimension, and Groups EDG and EDW began with form. During the postshift problem, color was the relevant dimension for all groups.

Subjects. Forty undergraduates from the introductory psychology course. who met the same criteria as subjects in Experiment I, were randomly assigned to the four groups. One subject was a nonsolver and was replaced by another student.

\section{Results}

Transformation of raw scores was again necessary. Since analyses of variance of the transformed scores on trials and errors yielded the same results, only analyses of the transformed trials data are reported.

Preshift mean trials to criterion for Groups IDG, EDG, IDW, and EDW were .873, .914, .785, and .867, respectively. Corresponding postshift means were $.464 .1 .173, .438$, and 1.111 . As in Experiment I, the data indicate that there were "savings" from preshift to postshift for the ID groups but not for the ED groups, and that both ID groups learned the postshift task faster than either of the ED groups.

An analysis of variance of the preshift scores indicated that the main effects and the interaction were nonsignificant (all Fs $<1$ ). The analysis of the postshift scores revealed that the Shift effect was reliable, $\mathrm{F}(1,36)=20.21, \mathrm{p}<.01$, indicating that an ID shift was learned faster than an ED shift. The Stimulus effect and the Shift by Stimulus interaction were not significant (both Fs $<1$ ).

In checking for stimulus generalization directly, it was found, contrary to the generalization hypothesis. that more subjects in Group IDW made correct stimulus pairings on the first trial of the postshift than did subjects in Group IDG. The respective proportions were $80 \%$ and $60 \%$. The difference between the two proportions was not reliable, $\mathrm{z}=.98, \mathrm{p}>.20$. No subjects in either group made consecutive errors on the first two trials of the postshift.

\section{DISCUSSION}

Regardless of the class of stimuli used, geometric or symbolic. ID shifts were learned more rapidly than ED shifts. In addition. original learning with both types of stimuli was found to be comparable. The finding of ID shift superiority with a total change design and word stimuli replicates LeBow and Tritt (1971) and Slamecka (1969), and is consistent with the vast majority of prior concept shift studies which did not employ these procedures. There was no evidence to support the hypothesis that color-relevant ID shifts with geometric stimuli were facilitated by a nonmediational process such as stimulus generalization (Slamecka, 1968). The results lend further support to a two-stage mediational interpretation of discriminative learning (Kendler \& Kendler, 1962).

\section{REFERENCE NOTE}

1. Gersten, C. D. Comparison of intradimensional and extradimensional shifts using a total change design. Unpublished manuscript. Arizona State University. 1971.

\section{REFERENCES}

Buss, A. H. Rigidity as a function of reversal and nonreversal shifts in the learning of successive discrimination. Journal of Experimental Psychology, 1953, 45, 75-81.

Eimas, P. E. Effects of overtraining and age on intradimensional and extradimensional shifts in children. Journal of Experimental Child Psychology, 1966, 3, 348-355.

Kendler, H. H., \& D'Amato, M. F. A comparison of reversal shifts and nonreversal shifts in human concept formation behavior. Journal of Experimental Psychology, 1955, 49. 165-174.

KendleR, H. H., \& Kendler, T. S. Vertical and horizontal processes in problem solving. Psychological Review, 1962. 69. 1-16.

LEBow, M. D..\& TRITT, J. Intradimensional and extradimensional shifts using a total change design with word stimuli. Psychonomic Science, 1971, 22, 325-327.

Slamecka. N. J. A methodological analysis of shift paradigms in human discrimination learning. Psychological Bulletin. 1968. 69. 423-438.

Slamecka, N. J. An improved discrimination-shift design. Psychonomic Science, 1969, 17. 89.

(Received for publication February 3, 1976.) 